Revista Calidad en la Educación Superior

Programa de Autoevaluación Académica

Universidad Estatal a Distancia, Costa Rica

ISSN 1659-4703

revistacalidad@uned.ac.cr

\title{
CONCEPTUALIZANDO LAS CONSIGNAS COMO PARTE DE LAS ACTIVIDADES DE UN CURSO VIRTUAL: UNA EXPERIENCIA BASADA EN LOS PROCESOS DE CAPACITACIÓN EN LA UNED
}

CONCEPTUALIZING THE INSTRUCTIONS AS A PART OF VIRTUAL CLASS ACTIVITIES: AN EXPERIENCE BASED ON THE PROCESS OF TRAINING AT UNED.

Julia Pérez Chaverri ${ }^{1}$ jperez@uned.ac.cr

Universidad Estatal a Distancia, Costa Rica

Volumen 6, Número 2

Noviembre 2015

Pp. $136-163$

Recibido: 4 de agosto, 2015

Aprobado: 26 de setiembre, 2015

\footnotetext{
${ }^{1}$ Julia Pérez Chaverri, M.Ed, Centro de Capacitación en Educación a Distancia, Universidad Estatal a Distancia, Costa Rica. Correo electrónico: jperez@uned.ac.cr
} 


\section{Resumen}

Este artículo pretende mostrar la sistematización de algunos conocimientos y experiencias del Centro de Capacitación en Educación a Distancia (CECED), en relación con qué son y cómo se elaboran las consignas, las cuales generan los procesos de comunicación sincrónicos y asincrónicos en los cursos desarrollados en las plataformas virtuales institucionales. Dado que dichos entornos permiten ampliar, potenciar y maximizar los procesos formativos; se han estructurado diferentes estrategias de comunicación, interacción y mediación que promueven el aprendizaje autónomo y autorregulado de las personas participantes. Como parte de esa estrategia, se elaboran y se usan las consignas para cada actividad de cada curso.

Palabras clave: capacitación, consignas, comunicación asincrónica, comunicación sincrónica, estrategia de aprendizaje, autonomía, autorregulación, cursos virtuales.

\section{Abstract}

This article shows the systematization of the knowledge and experience of the Centro de Capacitación en Educación a Distancia (CECED), in relation to the meaning and the process of elaborating the instructions, which generate the processes of synchronous and asynchronous communication in the courses developed in the institutional virtual platforms. Given that these environments can extend, enhance and maximize the learning processes; different strategies of communication, interaction and mediation that promote independent learning and self-regulating of the participants have been structured. As part of that strategy, the instructions for each activity of each course are elaborated and used.

Keywords: training, instructions, synchronous communication, asynchronous communication, learning strategy, autonomy, self-regulation, online courses.

\section{Introducción}

En 2015, el Centro de Capacitación en Educación a Distancia (CECED) de la Universidad Estatal a Distancia (UNED) cumplió 15 años de haber sido creado, lo cual lleva a reflexionar sobre cómo se han realizado todos los procesos de capacitación y cómo estos han evolucionado. Es importante recalcar que en sus inicios, el centro trabajaba con una metodología más presencial que a distancia, y por ende, la cantidad y la variedad de actividades de capacitación eran muy reducidas (Pérez, 2011). 
Con la llegada de las plataformas virtuales a la UNED, el CECED poco a poco fue implementando procesos de capacitación más a distancia que presenciales, lo cual permitió ir acumulando conocimientos, experiencias, y aciertos y desaciertos sobre cómo diseñar estrategias de enseñanza-aprendizaje para ser implementadas en el aula virtual, especialmente, mediante las distintas herramientas de comunicación e interacción (foro, chat, correo, tarea, cuestionario, etc.).

Como se indica en el Modelo Pedagógico de la UNED, se hace necesario encontrar metodologías apropiadas para llevar a cabo procesos de educación a distancia, en los que el estudiantado protagonice su propia construcción del conocimiento durante el proceso de enseñanza-aprendizaje. Es por eso que "se requiere aprovechar todo el potencial de las tecnologías al servicio de una formación más autónoma" (UNED, 2004, p.11), y la capacitación no escapa a estos postulados.

En este sentido, el CECED, además de capacitar sobre el modelo pedagógico institucional, también debe modelar sobre cómo pueden llevarse a cabo diversos procesos de enseñanza-aprendizaje apoyados en recursos tecnológicos, como las plataformas virtuales. Una de las temáticas que atañen a esta labor tiene que ver con cómo se logra establecer una comunicación e interacción sincrónica y asincrónica apropiadas para un entorno virtual.

Una de las formas de llevar a cabo lo anterior es mediante las consignas, las cuales le indican a la persona participante o al estudiantado, de manera sencilla y organizada, las indicaciones de lo que se espera obtener durante y al final de cada una de las actividades que forman parte de las estrategias de aprendizaje planificadas para cada asignatura, curso o programa. De esta manera, es importante que las consignas estén elaboradas de la forma óptima, pues así, podrán promover procesos de aprendizajes autónomos y autorregulados. 
Tal como se indica en el documento Cómo ofertar y diseñar cursos en línea, "se espera que el docente tutor, por medio de los foros, consignas de trabajo, los procesos planificados y los recursos provoque la construcción del conocimiento en los estudiantes" (PAL y PACE, 2010, p.37). Es por eso que, como parte de la acción docente, se debe organizar el tiempo y espacio para que en la consigna se señalen las fechas de inicio y cierre de cada una de las actividades. Así, el estudiantado o las personas participantes pueden decidir cuándo iniciar o continuar con la construcción y reconstrucción de conocimientos; es decir, cuándo iniciar o terminar la actividad. En consecuencia, las personas no requieren estar conectadas en el mismo momento y lugar para llevar a cabo el proceso de interacción con el entorno virtual, los materiales didácticos, el facilitador y el resto del grupo (UNED, 2004).

Asimismo, desde hace tres años, el CECED ha realizado diversas capacitaciones (taller Elaboración de consignas para actividades de cursos en línea y curso Organización y diseño de cursos en línea, por ejemplo), con el fin de mejorar el contenido y la estructura de las consignas que se colocan en las actividades de los cursos virtuales. Por esta razón, mediante este artículo, se pretende sistematizar algunos conocimientos y experiencias sobre qué es y cómo se elaboran las consignas que son parte de las actividades que se desarrollan en las plataformas virtuales y que permiten procesos de comunicación sincrónicos (al mismo tiempo) y asincrónicos (en diferentes momentos). De tal forma que, a través de estas, se promueva el aprendizaje autónomo y autorregulado de los participantes. 


\section{Acercamiento teórico}

Sólo hay una pequeña parte del universo de la que sabrás con certeza que puede ser mejorada, y esa parte eres tú.

AldousHuxley

En la UNED, el equipo docente debe trabajar de manera coordinada para diseñar, actualizar y ofertar cada curso, asignatura o programa. De la misma manera, la construcción de las consignas que presentan las actividades no es una tarea docente que se pueda realizar de manera aislada y descontextualizada, ya que esta debe tener completa relación con la estrategia de aprendizaje, el instrumento de evaluación y la herramienta tecnológica que se habilita dentro del aula virtual (Amador y Pérez, 2015).

Asimismo, la construcción de consignas es una habilidad de comunicación escrita que el equipo docente requiere desarrollar o perfeccionar, y es por eso que el CECED ha dedicado varios procesos de capacitación para trabajar esta área o temática. Al respecto, según Hernández (s.f.), la función tutorial se concreta cuando uno o más docentes llevan a cabo un proceso de orientación y acompañamiento para que cada estudiante alcance los objetivos propuestos mediante:

- La comprensión de los contenidos

- La interpretación de las descripciones procedimentales para la realización de trabajo

- La realización de ejercicios o autoevaluaciones

- La aclaración precisa de las dudas

Es por eso que sin una buena consigna, nada de lo anterior podría llevarse a cabo en un entorno virtual, o al menos requeriría más tiempo y esfuerzo del necesario por parte del grupo de estudiantes o participantes. 


\section{¿Qué es una consigna?}

La autonomía es un requisito para la eficacia Jack Lang

En el contexto de la UNED se requiere una reflexión permanente sobre cómo los equipos docentes se comunican de manera sincrónica y asincrónica con el estudiantado, cuando un proceso de enseñanza- aprendizaje se realiza a distancia y especialmente, cuando este se da en una plataforma virtual. En este sentido, las actividades y las diferentes herramientas tecnológicas generan oportunidades de interacción en diferentes momentos, lugares y direcciones. Por lo tanto, una consigna puede hacer que la persona que aprende entre en contacto con:

\section{Figura 1. Contexto de una consigna}

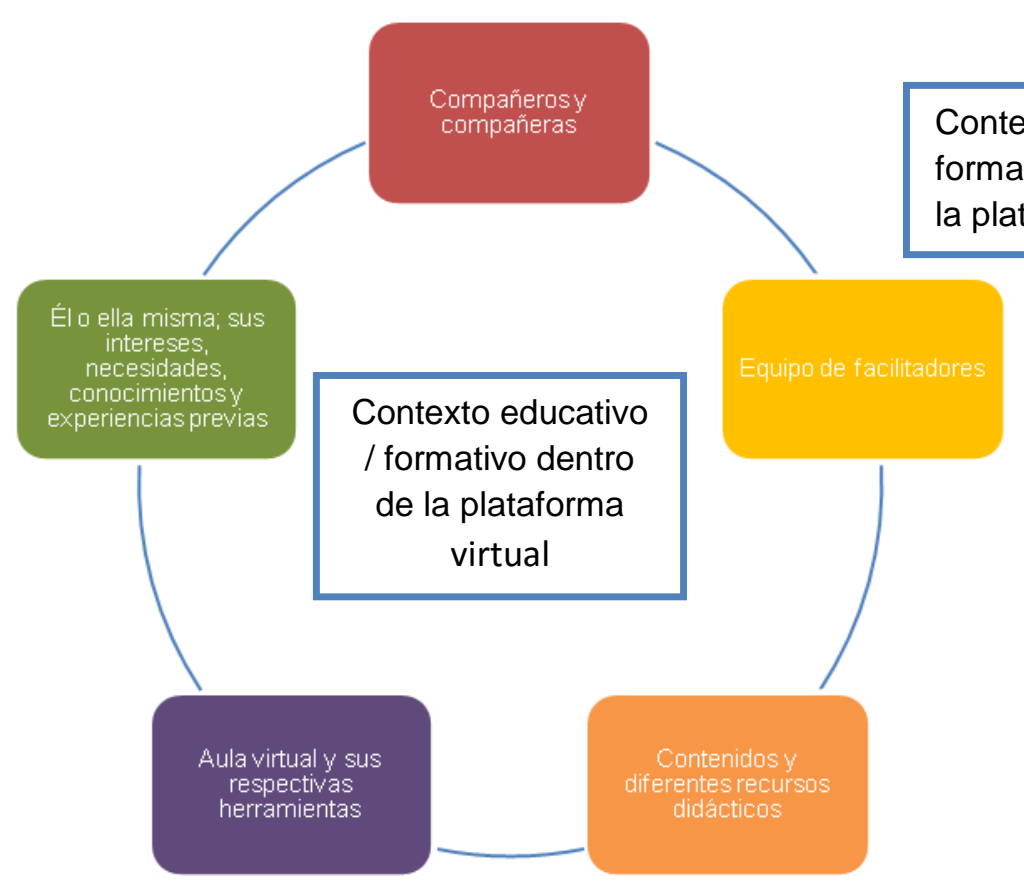

Fuente: Elaboración propia

En relación con lo anterior, García y Perera (2007) indican que la comunicación con fines educativos es un proceso que tradicionalmente se enmarcaba dentro de un ambiente físico en el que interactúan diversas personas; sin embargo, conceptualizar este proceso hoy es todo un reto, pues los diferentes recursos 
tecnológicos permiten que la práctica comunicativa sea un fenómeno aún más complejo. De ahí que podemos hablar de interacción dentro de entornos virtuales, de comunicación sincrónica y asincrónica, y de uso de diferentes códigos de lenguaje (en los que predomina el lenguaje escrito).

Este tipo de comunicación, cuando se lleva a cabo dentro de un entorno virtual de aprendizaje, requiere de elementos estratégicos que orienten y promuevan el proceso de construcción y reconstrucción del conocimiento que realiza el grupo de participantes. Uno de los elementos estratégicos es precisamente la consigna, la cual debe construirse previamente para garantizar que el contenido o información cumpla el propósito para lo cual fue creada. Tal y como lo indica Meneses (2012), la información que es emitida por una persona, si utiliza código común, puede generar una determinada reacción en otra persona, siempre y cuando este código logre realizar la significación esperada.

Partiendo desde lo más sencillo, una consigna es una "orden que da... a los subordinados o afiliados." Son las "órdenes que se dan a quien manda un puesto" (Real Academia Española, 2007). Esta definición hace hincapié en los "mandatos o indicaciones"; por lo tanto, existe una persona que da las indicaciones y otra(s) que las acata(n). Lo anterior es así precisamente porque la consigna como parte de una actividad que se desarrolla en un entorno virtual- contiene esa información concreta y directa que le solicita al participante o estudiante una evidencia, un producto, un resultado (observable, explícito y verificable) que demuestre el aprendizaje alcanzado o las habilidades desarrolladas.

Ahora bien, en términos más amplios y desde el contexto de capacitación desarrollada en los últimos años en esta universidad, una consigna se puede visualizar también como se muestra en la tabla 1 (CECED, 2013). 
Tabla 1. Percepción de la consigna

\begin{tabular}{|c|c|}
\hline Desde el punto de vista docente & $\begin{array}{c}\text { Desde el punto de vista de la } \\
\text { persona participante/estudiante }\end{array}$ \\
\hline - Conjunto de indicaciones claras & - Mensaje inicial que aparece en la \\
y concretas que se organizan en & herramienta virtual y que explica \\
secuencia para que la persona & la secuencia de pasos que se \\
participante o estudiante realice & deben seguir para lograr el \\
una actividad que es parte de la & producto o evidencia de la \\
estrategia de aprendizaje. De ahí & actividad en un plazo \\
que el equipo docente realiza un & determinado. \\
análisis previo de los pasos, & Secuencia de pasos que permite \\
etapas o procesos que debe & desarrollar y demostrar \\
llevar a cabo la persona para & autonomía y autorregulación. \\
lograr un determinado resultado. & Además, da insumos para \\
& realizar un proceso de \\
& autoevaluación constante. \\
& Comunicación oficial y formal \\
& que da el facilitador para indicar \\
& actividad específica. \\
&
\end{tabular}

Elaboración propia a partir de CECED (2013)

Ahora bien, en un contexto educativo o formativo presencial, la consigna es la explicación que da un profesor o profesora en el aula cuando le solicita al estudiantado realizar alguna actividad o proceso para llegar a una meta u objetivo determinado, una vez terminado el tiempo establecido para ello. Sin embargo, en la presencialidad suelen darse situaciones como las siguientes: 
El profesor o profesora explica:

- Con exceso de detalles, y al final no todo el estudiantado recuerda toda la información

- Muy rápido, y no todo el grupo logra seguir el ritmo de la explicación

- De manera escueta, y al final el estudiantado tiene muchas dudas y vacíos de información

- Varias veces, pero no necesariamente explica lo mismo, lo cual puede generar cierta incertidumbre en el grupo de estudiantes.

Algunos(as) de los(as) estudiantes:

- Están prestando atención; otros(as) prestan atención a veces, y los(as) demás no estás prestando nada de atención.

- Recuerdan lo que se les solicitó, pero no necesariamente pueden repetirlo con las mismas palabras o detalles.

- No comprenden la información que se les da para lograr la meta o el objetivo propuesto, y prácticamente pasan haciendo preguntas durante todo el proceso. El que puedan terminar el trabajo solicitado depende de la prontitud y la calidad de las respuestas que les den.

- Trabajan con rapidez y terminan antes, mientras que a otros(as) no les da tiempo de hacer el mismo procedimiento en el mismo tiempo.

- Entre otras situaciones.

En un contexto educativo o formativo a distancia, mientras mejor construidas estén las consignas, más se evitará que se presenten situaciones como las anteriores. En este sentido, de acuerdo con CECED (2013) es importante que estas cumplan al menos las siguientes condiciones:

- Deben establecer y conservar el buen trato y la cordialidad en la expresión escrita, y deben resaltar la parte humana, especialmente porque se está trabajando en un entorno virtual. Así, las consignas deben construirse como 
si las personas estudiantes o participantes estuvieran de frente al docente. (Millán, s.f).

- Deben motivar a la persona participante o estudiante, ya que es sobre la base de la motivación que puede establecerse el aprendizaje (UNED, 2004, p. 21). Por ejemplo, lo importante no es resaltar cuántos puntos se pueden ganar o perder con cada actividad, sino más bien enfatizar en todos los beneficios que se obtienen al apropiarse de ciertos conocimientos 0 al desarrollar ciertas habilidades.

- Deben lograr un texto escrito claro y comprensible, sin errores gramaticales, ortográficos o de digitación. Al respecto, hay que tener claro que no es lo mismo dar una explicación de manera oral a hacerlo de forma escrita (Millán s.f, p. 5): en una situación presencial, es común abusar de la cantidad de palabras o incluso de la complejidad de estas; además, si un mensaje no es comprendido, el o la docente puede tomarse el tiempo para dar más detalles o una nueva explicación. En cambio, en un entorno virtual, se debe buscar la manera más sencilla y completa de indicar los pasos a seguir, sin caer en reiteraciones y sin brindar exceso de información.

- Deben reconocer qué se puede solicitar y hasta dónde es factible exigir a las personas participantes o estudiantes. Al respecto, no todas las personas poseen las mismas condiciones de estudio ni las mismas habilidades, experiencias y conocimientos previos. Por esto, es importante que al inicio del curso o asignatura exista algún espacio para conocer características básicas del grupo; por ejemplo, dónde viven, cuáles son los horarios de estudio o trabajo, etc. Según el modelo pedagógico de la UNED, un principio de la educación de adultos es el conocimiento previo y debe tenerse en cuenta especialmente para el establecimiento de las secuencias de aprendizaje (2004, p. 22).

- Deben escribirse y leerse como si fueran parte de una conversación académica; al mismo tiempo que debe evitarse el redactarlas como un regaño, un sermón o un conjunto de imposiciones. 
La ventaja de construir consignas desde la modalidad a distancia es que la persona docente a cargo puede revisarlas y analizarlas antes de colocarlas en el espacio respectivo dentro del entorno virtual del curso, asignatura o programa. Esto permite minimizar los errores y verificar que haya congruencia con la estrategia de aprendizaje e incluso con los criterios de evaluación, independientemente de si la evaluación es cualitativa o cuantitativa (Amador y Pérez, 2015).

\section{¿Qué elementos componen una consigna?}

El tema de las consignas se ha desarrollado en el CECED en el taller Elaboración de consignas para actividades de cursos en línea y en el curso Organización y Diseño de Cursos en Línea. De acuerdo con tales experiencias, se ha logrado determinar que la consigna debe contener cierto tipo de información y ciertos componentes, los cuales se presentan a continuación:

- El saludo: debe ser cordial y respetuoso, ya que este inicia la conversación asincrónica con el grupo de participantes o estudiantes.

- Qué se logrará: la consigna debe hacer una breve alusión sobre la relación que tiene la actividad propuesta con el objetivo de aprendizaje. Esto permite que la persona encuentre relevancia y conexión entre la actividad específica y todo el curso, asignatura o programa.

- Qué hacer: implica una previsión y detalle de lo que la persona estudiante o participante debe hacer. Por ejemplo, elaborar un ensayo, un texto paralelo, un avance de una investigación, un diario reflexivo, un mapa mental, un mapa conceptual, etc. La elección del qué hacer depende de los objetivos de aprendizaje y contenidos que se deben desarrollar en el curso, asignatura o programa. En este sentido, si la actividad a realizar es muy compleja y extensa, es mejor que el docente realice una consigna para cada parte, etapa o avance del trabajo solicitado.

- Cómo se hará: la consigna incluye la secuencia de pasos que la persona estudiante o participante debe seguir. Al igual que como ocurre con el punto 
anterior, esta secuencia tendrá que ver, en gran parte, con el tipo de actividad que debe realizarse. Es importante tener presente que para que el o la docente pueda explicar de manera óptima cómo debe llevarse a cabo la actividad, es necesario que este no solo tenga una gran habilidad para comunicarse de manera escrita, sino también mucha experiencia y conocimiento sobre la actividad en sí misma. Por ejemplo, si un docente no domina la estructura de un mapa conceptual, difícilmente podrá indicar los pasos a seguir para elaborar uno, y probablemente, tampoco tendrá los conocimientos necesarios para construir los criterios de evaluación, sean cualitativos o cuantitativos. Otros elementos a tomar en cuenta esta parte de la consigna es indicar el tipo de interacción que se requiere como parte del proceso para llegar al determinado producto o evidencia; es decir, si se hará de manera individual, en parejas, grupalmente o incluso, si se organizará por roles. Asimismo, deben incluirse otros aspectos, tales como la extensión, el tipo de letra, el interlineado, etc. Igualmente, si se solicita un formato, machote, cuadro o plantilla especial, se debe adjuntar en esta parte de la consigna.

- Dónde se hará: en esta parte se hace referencia al lugar donde deberá realizarse la actividad o qué parte de ella. Esto indicará si se hará únicamente dentro del entorno virtual y mediante una herramienta específica; o bien, si se llevará a cabo en algún lugar físico-geográfico y luego deberá enviarse un reporte, informe o evidencia, a través de alguna de las herramientas que posee el entorno virtual.

- Cuándo se hará: se refiere a los plazos de ejecución, y en esta sección no solo se indica en qué fecha y hora debe entregarse el trabajo solicitado, sino también el tiempo disponible que se posee para llevarlo a cabo. Este aspecto se define de acuerdo con la complejidad y extensión de la actividad y la organización general del curso, asignatura o programa. Es importante recordar que los tiempos dentro de un proceso educativo o formativo que se desarrolla en una plataforma virtual no se manejan igual que los tiempos de 
los procesos presenciales, pues la asincronía es más frecuente que la sincronía. En este sentido, los plazos deben favorecer a la persona estudiante o participante y no se organizan en función de los horarios del equipo docente.

- Despedida y firma: al igual que el saludo, la despedida debe ser cordial y amigable. Incluir estos dos elementos hace que la consigna sea más personal.

\section{Acercamiento práctico}

\section{¿De dónde proviene la información que se plantea en la consigna?}

Elaborar un consigna es una tarea concreta que se lleva a cabo cuando se planifica una oferta académica que se desarrollará en un entorno virtual; sin embargo, es una tarea compleja pues quien la construye no puede perder de vista otros elementos que componen ese curso, asignatura o programa y cómo estos se influyen mutuamente. No tomarlos en cuenta podría provocar que el proceso de comunicación e interacción tenga la dirección equivocada. Algunos de esos elementos que van más allá de la consigna se puede visualizar en la figura 2.

\section{Figura 2. Información que fundamenta la consigna}

\section{De la matriz a la consigna}

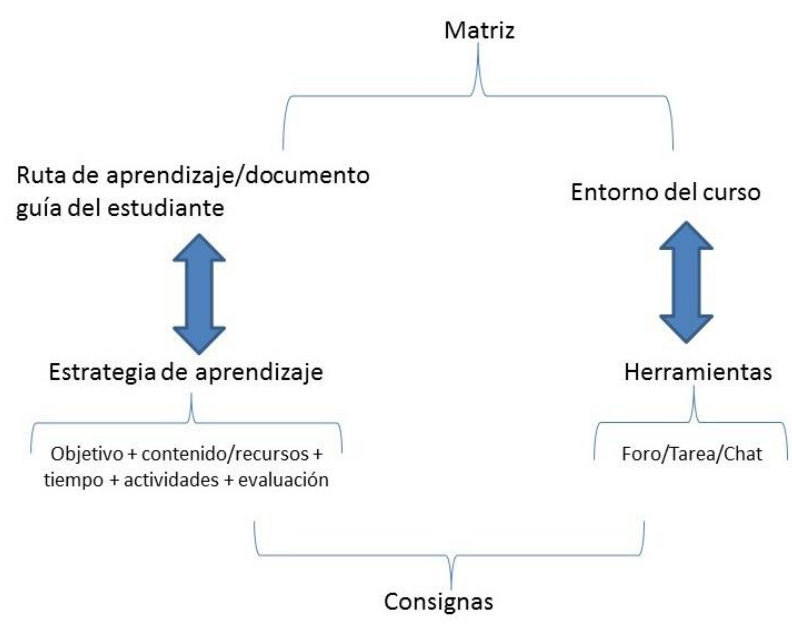

Fuente: Elaboración propia 
- Matriz de programación: es un documento que se utiliza en la UNED y se elabora con base en el diseño curricular. Permite planificar de manera detallada, concreta y dosificada todo el curso/asignatura a distancia cuya oferta se desarrollará a través de la plataforma institucional. Esta planificación previa (uno o dos cuatrimestres antes) es fundamental, porque ofrece la siguiente información y organización: unidades o módulos que conforman el curso/asignatura, cantidad de semanas que dura cada unidad o módulo, fechas de inicio y cierre, objetivos y contenidos que se trabajarán por unidad o módulo, recursos y materiales que deberá utilizar la persona estudiante de manera obligatoria y complementaria, las actividades que se realizarán en cada unidad o módulo y que permiten el alcance de los objetivos de aprendizaje, y el tipo de evaluación que se realizará en cada unidad o módulo (UNED, 2010).

- Estrategia de aprendizaje: es un conjunto de acciones, pasos o secuencias cognitivas, afectivas o motoras que se realizan de forma consciente y planificada para la consecución de un objetivo de aprendizaje o para la resolución de una situación de aprendizaje (Programa de Apoyo Curricular y Evaluación de los Aprendizajes, 2012, p. 38). Por lo tanto, de la estrategia se extraen las actividades, las cuales orientan el tipo de herramienta tecnológica que se debe seleccionar y habilitar dentro del entorno virtual.

- Estrategia de evaluación: es el conjunto de acciones que realiza la persona para evidenciar el logro de los objetivos de aprendizaje durante el curso o asignatura. Esta estrategia está completamente relacionada con la de aprendizaje, al punto que ambas se fusionan, se nutren y se fundamentan. Tal como se indica en el Plan Académico 2015-2017, se espera que la evaluación sirva para aprender, y el aprender sirva para evaluar. La manera en la que se diseña la estrategia de aprendizaje es lo que posibilita que la persona (facilitador (a) / estudiante) tenga mayor o menor participación y compromiso con la evaluación. Sin embargo, si se diseña la propuesta evaluativa con una visión tradicional y conductista, probablemente en la 
matriz se encuentre una secuencia de actividades para que la persona "estudiante estudie" y otra secuencia de actividades las cuales serán "calificadas por el o la docente".

- Técnica de evaluación:

Una técnica de evaluación se define como un conjunto de operaciones que el estudiantado debe realizar para construir un producto o evidencia de aprendizaje. A partir de ella, se puede evaluar un procedimiento y su resultado. Al proponerlas, se debe tomar en cuenta el objetivo de aprendizaje, el contenido de estudio, la cantidad de estudiantes, el tiempo disponible para alcanzar el objetivo, la herramienta virtual de la cual se dispone y el conocimiento de la técnica en sí misma por parte de la persona facilitadora (Amador y Pérez, 2015, p. 20).

El tipo de técnica de evaluación se seleccionará y se utilizará de acuerdo con la manera en la que se diseña la estrategia de aprendizaje y evaluación. También, depende del conocimiento y dominio que tenga el equipo docente sobre dicha técnica, de manera que la experiencia que se tenga sobre la técnica seleccionada permitirá extraer o sintetizar la información básica que fundamentará la consigna y el instrumento de evaluación.

- Instrumento de evaluación: recurso de apoyo en el que se registran los conocimientos, habilidades y destrezas que desarrolla la persona durante la aplicación de una estrategia o técnica de evaluación. Pueden ser listas de cotejo, escalas de calificación, matrices de valoración, etc. Se diseñan para generar procesos de evaluación más objetivos, regulados o autorregulados (PACE, 2012). Además, el instrumento indica cuáles son los criterios con los que se va a evaluar la actividad o tarea, así como los requisitos que debe cumplir este producto, evidencia o resultado, lo cual debe 
especificarse dentro de la consigna. No se puede evaluar un aspecto que no se solicite dentro de la consigna.

- Herramienta tecnológica: las herramientas pueden facilitar el proceso de comunicación sincrónico o asincrónico, lo cual implica que es en diferente hora y lugar. (PACE, 2012). Dichos procesos de comunicación e interacción pueden darse entre facilitador(a)-estudiante, estudiante- facilitador(a), facilitador(a)-estudiantes, estudiantes- facilitador(a), estudiante-estudiante, estudiante-estudiantes, estudiantes- estudiante, estudiantes-estudiantes. Las herramientas permiten esa interrelación en múltiples direcciones, pero por sí solas no generan ni comunicación ni interacción y mucho menos aprendizaje.

- Actividades: según Delgadillo (s.f.), "son ejercicios o supuestos prácticos que pretenden que la persona estudiante no se limite a memorizar, sino que esté constantemente aplicando los conocimientos con la finalidad de que los convierta en algo operativo y dinámico" (p. 62). Cabe resaltar que no necesariamente todas las actividades que debe realizar la persona se hacen dentro del aula virtual, aunque todas deben o deberían ser parte de la estrategia de aprendizaje. Es decir, una actividad por sí sola no es una estrategia de aprendizaje pero dentro de una estrategia de aprendizaje podemos encontrar diferentes actividades.

Cuando se tiene elaborada la consigna, esta se coloca dentro de la herramienta tecnológica que se habilitó para ello en la plataforma virtual, pero antes se debe tener organizado ese espacio dentro de la plataforma. Tal y como se muestra en la figura 3, la organización permite que el curso, asignatura o programa cuenten con las unidades o módulos y estos, con sus respectivos recursos y actividades. 
Figura 3. Esquema de la unidad 0 dentro de un aula virtual

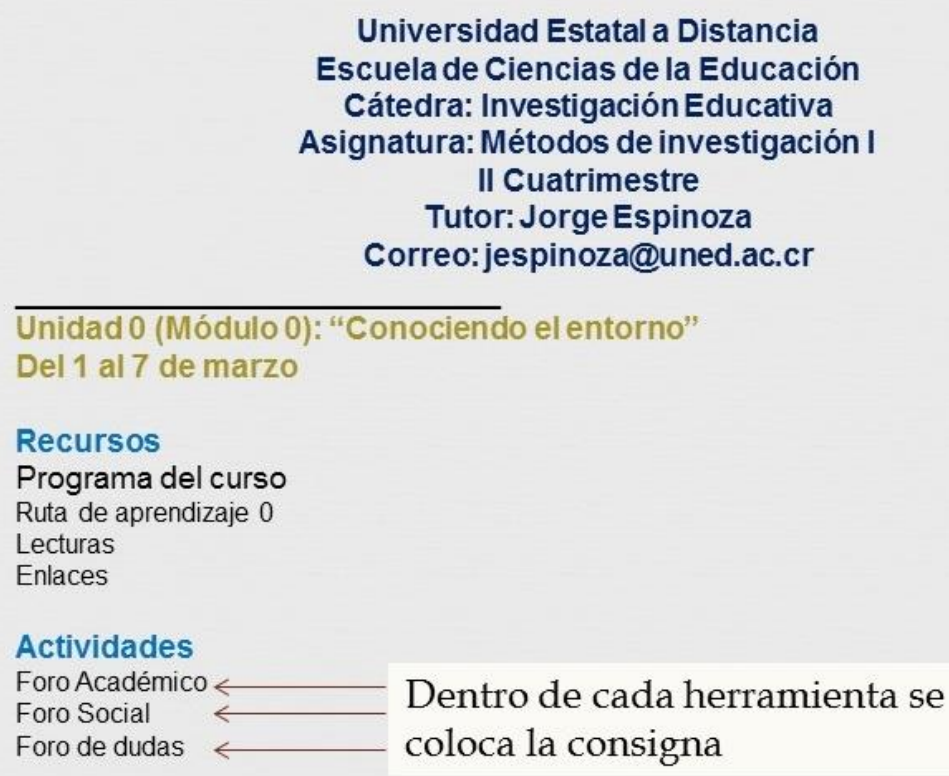

Fuente: Elaboración propia

\section{Ejemplos de consignas}

En este subapartado, se incluyen algunos ejemplos de consignas que se colocan en los diferentes espacios del aula virtual del curso Organización y diseño de cursos en línea.

\section{Ejemplo 1: Consigna que aparece en el foro académico de la Unidad 0 del curso:}

¡Buenos días, gente! ¿Cómo están?:

Es un placer reunirnos en este espacio para compartir algunos conocimientos y normas de participación en los foros, pues esto nos permitirá comunicarnos e interactuar mucho mejor, no solo en este foro, sino durante todo el curso. Así que:

1. Revisen los contenidos la unidad 0. 


\section{Lean el documento sobre Normas de participación en los foros.}

3. Conteste la siguiente pregunta generadora con fundamento en la lectura y en su propia experiencia personal: ¿Cómo y por qué debemos comunicarnos en los foros?

4. Coloquen su respuesta usando la opción "Responder" que aparece en este mensaje.

5. Lean las respuestas del grupo.

6. Comenten al menos 2 participaciones realizadas por sus compañeros y compañeras usando la opción "Responder" que aparece en el mensaje de la persona seleccionada.

7. Apliquen las normas de participación que se indicaron en la lectura cada vez que realice un aporte.

Este foro se autoevaluará de manera cualitativa con la rúbrica que se encuentra en el documento llamado Ruta de aprendizaje ubicado en la sección de Recursos, y estará disponible del 1 de febrero a las 10:00 a.m. al 7 de febrero a las 11:00 p.m.

iA trabajar, mucho compromiso y entusiasmo!

Roberto

Ejemplo 2: Consigna que aparece en foro 2 de la unidad 3 del curso:

¡Hola mis queridos amigos y amigas del grupo 1 (Ana, Juan, Pedro, Mónica)!:

En esta ocasión, ustedes se encargarán de coevaluar la propuesta del curso que cada quien está elaborando, lo cual les permitirá aprender de los demás y mejorar sus propios diseños. Para eso:

1. Lean los contenidos de la unidad 3 que se encuentran en la sección de Recursos.

2. Adjunten la matriz de programación con la que han venido trabajando en una línea nueva de discusión. Para esto, utilicen la opción "Colocar nuevo tema de discusión aquí". 
3. Revisen las matrices de los miembros del equipo.

4. Comenten el trabajo de cada compañero o compañera. Para esto, utilicen la opción "Responder" que se encuentra dentro de la línea de discusión que cada persona abrió.

5. Lean los comentarios recibidos.

6. Actualicen la matriz de programación con base en la realimentación recibida por los integrantes del equipo.

7. Suban la propuesta mejorada del curso dentro de la misma línea de discusión que abrió al inicio, usando la opción "Responder".

Esta actividad se coevaluará de manera cualitativa con base en la rúbrica que se encuentra en la Ruta de aprendizaje 3, y estará disponible del 7 de marzo a las 10:00 a.m. al 14 de marzo a las 11:00 p.m.

¡Todos para uno y uno para todos!

Roberto

Ejemplo 3: Consigna que aparece en la tarea 1 de la Unidad 1 del curso:

Estimado grupo:

Esta tarea nos permitirá ir transformando un curso a distancia tradicional en uno $100 \%$ virtual mediante la elaboración de la matriz de programación. Así que:

1. Lean los contenidos de la unidad que se encuentran en la sección de Recursos.

2. Lean el diseño o programa del curso que va a virtualizar.

3. Descarguen la matriz de programación en la que debe organizar el curso.

4. Completen las 3 primeras columnas de la matriz que descargó con la información que se solicita en cada una y de acuerdo con el contenido del 


\section{diseño o programa del curso.}

5. Revisen el instrumento de evaluación con el que se calificará esta tarea.

6. Expongan sus consultas sobre esta tarea únicamente por el foro de dudas, en caso de ser necesario.

7. Envíen la matriz de programación con la información solicitada por esta misma herramienta.

Esta tarea tiene un valor de $10 \%$ y la fecha límite para la entrega es el 15 de febrero a las 11:00 p.m.

¡Manos a la obra!

Roberto

Como se dijo en párrafos anteriores, las consignas se fundamentan no solo en el tipo de herramienta, sino también en otro tipo de información proveniente de la estrategia de aprendizaje y la matriz de programación. Tal y como se muestra en la tabla 2, la consigna ofrece información sobre la actividad por realizar, como el procedimiento operativo a ejecutar, tipo comunicación e interacción, la manera en la que se llevará a cabo la evaluación, entre otras. 
Tabla 2. Datos relevantes de los ejemplos anteriores

\begin{tabular}{|c|c|c|c|}
\hline & Ejemplo 1 & Ejemplo 2 & Ejemplo 3 \\
\hline Herramienta & Foro & Foro & Tarea \\
\hline $\begin{array}{l}\text { Aspectos } \\
\text { operativos }\end{array}$ & $\begin{array}{l}\text { Tipo de foro } \\
\text { académico de } \\
\text { debate sencillo, por } \\
\text { lo que cada } \\
\text { persona coloca sus } \\
\text { aportes con el } \\
\text { botón "Responder". } \\
\text { Se habilita un solo } \\
\text { foro y todos pueden } \\
\text { ver los comentarios } \\
\text { de todos. }\end{array}$ & $\begin{array}{l}\text { Tipo de foro } \\
\text { académico de uso } \\
\text { general en el cual se } \\
\text { utiliza la apertura de } \\
\text { nuevas líneas de } \\
\text { discusión. No es lo } \\
\text { mismo abrir nuevos } \\
\text { hilos de } \\
\text { conversación que } \\
\text { responder a ese } \\
\text { hilo, pues en este } \\
\text { caso, el mensaje } \\
\text { quedará enlazado al } \\
\text { primero. } \\
\text { Se habilita un foro } \\
\text { para cada subgrupo. } \\
\text { Cada subgrupo tiene } \\
\text { acceso a la } \\
\text { información de los } \\
\text { otros subgrupos, } \\
\text { siempre y cuando el } \\
\text { docente permita la } \\
\text { visualización de los } \\
\text { otros foros. }\end{array}$ & $\begin{array}{l}\text { La persona puede } \\
\text { descargar archivos } \\
\text { que el docente haya } \\
\text { adjuntado, } \\
\text { especialmente si en } \\
\text { ese mismo formato } \\
\text { debe presentar la } \\
\text { tarea. Dicha } \\
\text { herramienta permite } \\
\text { que la persona } \\
\text { envíe ese } \\
\text { documento al } \\
\text { docente. }\end{array}$ \\
\hline Interacción & $\begin{array}{l}\text { General: todo el } \\
\text { grupo dentro del } \\
\text { mismo foro }\end{array}$ & En subgrupos & Solo con el docente \\
\hline Comunicación & Asincrónica & Asincrónica & $\begin{array}{l}\text { No es una } \\
\text { herramienta para } \\
\text { entablar } \\
\text { conversaciones, } \\
\text { pero de igual forma } \\
\text { la interacción es } \\
\text { asincrónica }\end{array}$ \\
\hline $\begin{array}{c}\text { Enfoque de la } \\
\text { evaluación }\end{array}$ & Cualitativa & Cualitativa & Cuantitativa \\
\hline $\begin{array}{c}\text { Rol del } \\
\text { evaluador }\end{array}$ & Autoevaluación & Coevaluación & Heteroevaluación \\
\hline
\end{tabular}

Fuente: Elaboración propia 


\section{Conclusiones}

Con base en lo indicado anteriormente, se puede decir que si existe una adecuada consigna que oriente la actividad que debe realizar la persona participante 0 estudiante, esta podrá:

- Leer los contenidos con un enfoque más preciso; es decir, será menos probable que divague en detalles innecesarios.

- Ejecutar los pasos del trabajo (producto, evidencia) solicitado con mayor organización, seguridad y confianza; o sea, con autonomía y autorregulación.

- Autoevaluar los pasos que ejecuta y retomar los aspectos que considere necesarios. En otras palabras, la consigna le da el punto de salida y el punto de llegada, dentro de algo más general llamado ruta o proceso de aprendizaje.

- Exponer consultas solo cuando sean realmente necesarias para lograr el aprendizaje o resultado esperado. Como parte de esto, también podrá colaborar con la aclaración de dudas cuando un compañero o compañera del grupo requiera ayuda.

- Orientar su proceso de aprendizaje, autorregularse y autoevaluarse. No obstante, si bien la consigna es un conjunto de indicaciones que debe seguir al pie de la letra, esto no quita la posibilidad de la autonomía en la ejecución de la actividad

Cuando el curso, asignatura o programa se desarrolla en un entorno virtual, la comunicación e interacción se planifican al igual que las estrategias de aprendizaje, de manera que la consigna es el resultado de esa planificación. En este sentido, la elaboración de consignas requiere de mucha reflexión y análisis de todos los elementos que componen el curso o asignatura.

En relación con esto, en el entorno que se habilita dentro de la plataforma virtual, se contará con diferentes herramientas que posibilitarán la comunicación y la 
interacción, siempre y cuando la selección de cada una de ellas sea adecuada para lograr los objetivos planteados en la estrategia de aprendizaje. Si se hace una mala selección de la herramienta o no se conocen las ventajas y las limitaciones de estas, aunque la consigna se redacte con todos los aspectos estructurales, esto no asegura que el proceso de comunicación, interacción o aprendizaje tendrá la fluidez y pertinencia requerida.

Asimismo, la elaboración de consignas requiere de una habilidad de comunicación escrita por parte del equipo docente, motivo por el cual es necesario elaborar varias versiones antes de lograr la propuesta más adecuada. Encontrar ese punto de eficiencia también depende del dominio que posee el facilitador o docente de los contenidos o disciplina, de la actividad o técnica, y del conocimiento del funcionamiento del entorno virtual y de las herramientas específicas que se usarán durante la estrategia de aprendizaje.

Por otro lado, una estrategia de aprendizaje puede estar compuesta de una o más actividades; por lo tanto, no se puede pretender que todas las actividades se van a llevar a cabo dentro de la misma herramienta ni que en una sola consigna pueden explicarse todas las actividades a la vez. En este sentido, cada consigna debe redactarse de tal forma que se comprenda de manera total, sin la necesidad de leer la consigna de otra actividad que conforma la estrategia de aprendizaje. La suma de todas las consignas evidencia la ruta que sigue la persona participante 0 estudiante, para construir y reconstruir los conocimientos que se disponen en cada unidad o módulo dentro del entorno virtual.

Otra de las ventajas de la elaboración de consignas para actividades que se implementarán en un entorno virtual es que estas le permiten al facilitador o profesor desarrollar un mayor dominio sobre la dinámica del curso, asignatura o programa. Encontrar la secuencia de pasos para llevar a cabo un producto o evidencia de aprendizaje requiere de mucho tiempo, análisis, reflexión y a veces, 
hasta de innovación; por lo tanto, la consigna no solo debe pensarse como un procedimiento para obtener un resultado visible y observable, sino que también debe contemplar el procesamiento cognitivo que debe realizar la persona participante o estudiante; es decir, la consigna no es solo para desarrollar "destrezas manuales". En este sentido, la elaboración de consignas es una muy buena práctica docente, aunque la actividad se desarrolle en procesos educativos o formativos completamente presenciales.

\section{Recomendaciones}

Seguidamente, se establece un grupo de recomendaciones que pueden seguirse:

- Redactar la consigna con la menor cantidad de palabras para brindar la mayor cantidad de información, lo cual implica que la persona que realiza la consigna debe tener capacidad de síntesis. La consigna es concreta pero no escueta.

- Redactar la consigna en función de la persona estudiante, no en función del equipo docente. Cuando esto no se toma en cuenta, se dejan pasos sin indicar, pues la persona que la elabora supone que hay información que se deduce de las indicaciones.

- Redactar la consigna como si la persona docente y la persona estudiante estuvieran frente a frente, de manera tal que cuando el estudiantado lea el mensaje, se visualice como si estuviera siendo parte de la conversación.

- Utilizar la misma estructura gramatical, ya sea con el verbo en tercera persona (lea, conteste, comente) o con el verbo en infinitivo (leer, contestar, comentar). Cuando se está tratando de redactar la consiga, es mejor que cada paso se escriba en una oración o frase, y que el verbo que representa la acción principal esté siempre al inicio. Además, es recomendable colocar cada acción en pasos separados; por ejemplo, dos verbos reflejan dos acciones distintas, lo cual significa que cada una requiere su momento para llevarse a cabo, aunque estas estén relacionadas. 
- Visualizar a la persona estudiante siguiendo cada paso de la consigna. Este ejercicio es fundamental para lograr una mejor estrategia comunicativa, ya que si la persona encargada de elaborar las consignas puede visualizar al estudiante llevando a cabo la actividad, podrá valorar qué tan claro o confuso es el mensaje; si está complicado o sencillo; si está en orden o no posee la secuencia requerida; si está completo o falto de información. Cuando se aplica esta recomendación, la persona debe realizar exactamente lo que indica la consigna para valorar si el producto solicitado contiene todo lo necesario para evidenciar el aprendizaje.

- Organizar los pasos en secuencia lógica, lo cual le permitirá al estudiantado llevar un control de lo que ha realizado y qué le falta por realizar. Además, esto le facilita autorregular el proceso que realiza para lograr el producto solicitado. En ese sentido, el orden de los pasos es muy importante, pues no es lo mismo indicar al estudiante que en el foro debe, por ejemplo:

\begin{tabular}{|l|l|}
\hline \multicolumn{1}{|c|}{ Opción a } & \multicolumn{1}{c|}{ Opción b } \\
\hline $\begin{array}{l}\text { 1. Leer los contenidos. } \\
\text { 2. Contestar la pregunta generadora. }\end{array}$ & $\begin{array}{l}\text { 1. Contestar la pregunta generadora. } \\
\text { 2. Leer los contenidos. }\end{array}$ \\
\hline
\end{tabular}

En el primer caso, se le podría evaluar a la persona que sus respuestas tengan el respectivo fundamento teórico, basado en las lecturas; mientras que en el segundo caso, la persona puede contestar sin ningún tipo de fundamento.

- Solicitar a otra persona que lea la consigna, ya que esto disminuye las incongruencias. Generalmente, una actividad se puede explicar de diferentes maneras, pero no necesariamente la persona docente lo hace de la manera más sencilla para la persona estudiante. Al respecto, se debe tener presente que los conocimientos, experiencias y habilidades del equipo docente no son iguales que los del grupo de estudiantes; por lo tanto, lo 
que esa persona percibe al leer la consigna puede ser diferente a la intención de quien la redactó.

- Realizar los pasos de la consigna a manera de prueba, de tal forma que los imprevistos sean mínimos. Mientras más clara tenga la consigna el equipo docente, más fácil le será encontrar incongruencias con el instrumento de evaluación. Si las hubiera, tendrá también una idea clara de cuál criterio se debe modificar, eliminar o incluir.

- Tener a la mano un diccionario. Las palabras que se utilizan de manera oral en la cotidianeidad no necesariamente se usan con el significado correcto. Asimismo, el uso del diccionario permite que se conozcan sinónimos que enriquecen el mensaje que se le da al estudiantado. Si se usa el vocabulario adecuado, esto promoverá una interpretación más objetiva en el estudiantado.

- Colocar un ejemplo de lo que se espera que realice el estudiantado. A veces, la actividad o el producto solicitado es tan complejo que explicar todos los pasos puede convertirse en un texto con exceso de información. Cuando esto sucede, se le debe indicar a la persona ver el ejemplo y basarse en este para llevar a cabo la tarea solicitada.

- Comparar la consigna con el instrumento de evaluación, independientemente de si se hace primero la consigna y luego el instrumento $\mathrm{o}$ al revés. Ambos elementos deben ser congruentes, por lo que si se actualiza o se mejora la consigna, debe revisarse el instrumento y viceversa. 


\section{Referencias}

Amador, O. y Pérez, J. (2015). Contenidos del curso Organización y diseño de cursos en línea, código 02-115. Unidad 3: Escribiendo el libreto: redacción de consignas y rutas de aprendizajes, Universidad Estatal a Distancia.

Centro de Capacitación en Educación a Distancia (2010). Contenidos del curso Enseñar y aprender en los entornos virtuales. Módulo 2: Estrategias de aprendizaje para la educación por medio de los entornos virtuales, Universidad Estatal a Distancia.

Centro de Capacitación en Educación a Distancia (2013). Materiales del taller Elaboración de consignas para actividades de cursos en línea, código 12213-02

Delgadillo, R. (s.f.). Actividades de aprendizaje como estrategia de enseñanza. El caso de tres cursos en línea. Centro de Enseñanza para Extranjeros, Universidad Nacional Autónoma de México. Recuperado de http://132.248.130.20/revistadecires/articulos/art12-4.pdf

García, C. y Perera, V. (2007) Comunicación y aprendizaje electrónico: la interacción didáctica en los nuevos espacios virtuales de aprendizaje. Recuperado de http://www.revistaeducacion.mec.es/re343/re343_17.pdf

Hernández, P. (s.f.). Funciones de la tutoría virtual. Recuperado de http://www.monografias.com/trabajos-pdf/funciones-tutoria-virtual/funcionestutoria-virtual.pdf

Loría, M. (2012). Comunicación oral y escrita. Guía de estudio. Recuperado de http://www.uned.ac.cr/ecsh/images/documentos/LitGrama/guiADIDActica709-2012-3.pdf

Millán, M. (Comp.) (s.f.). Comunicación escrita. Universidad de Londres. Recuperado http://www.uvirtual.edu.co/docudiseo/Dise\%C3\%B10\%20Grafico/AD/comunicacion_escrita.pdf

Programa de Apoyo Curricular y Evaluación de los Aprendizajes (2012). Glosario de términos. Universidad Estatal a Distancia. Recuperado de http://www.uned.ac.cr/academica/index.php/pace/inicio

Programa de Aprendizaje en Línea y Programa de Apoyo Curricular y Evaluación de los Aprendizajes (2010). Cómo diseñar y ofertar cursos en línea. Universidad Estatal a Distancia. 
Pérez, J. (2011). La capacitación a través de los entornos virtuales: una experiencia de desarrollo profesional en la UNED. Congreso Internacional de Nuevas tendencias en la formación permanente del profesorado, Barcelona. Recuperado de http://www.ub.edu/congresice/actes/2_rev.pdf

Real Academia Española (Ed.) (2007). Consigna. Diccionario de la Real Academia Española, (22a.ed.). Recuperado de http://lema.rae.es/drae/?val=consigna

Universidad Estatal a Distancia (2004). Modelo Pedagógico de la Universidad Estatal a Distancia. Aprobado por el Consejo Universitario, sesión № 1714, Artículo IV, inciso 3) del 9 de julio del 2004. Centro de Información y de Documentación y Recursos Bibliográficos (CIDREB). 